\title{
EXTENSION OF AUTOMORPHISMS OF SUBGROUPS
}

\author{
G. G. BASTOS \\ Departamento de Matemática, Universidade Federal do Ceará, Campus do Pici, \\ CEP 60455-760, Fortaleza, Ceará, Brazil \\ e-mail: ggbastos@ufc.br \\ E. JESPERS \\ Department of Mathematics, Vrije Universiteit Brussel, Pleinlaan 2, 1050 Brussel, Belgium \\ e-mail: efjesper@vub.ac.be \\ S. O. JURIAANS \\ Instituto de Matemática e Estatística, Universidade de São Paulo, \\ CP 66281, CEP 05311-970, São Paulo, Brazil \\ e-mail: ostanley@ime.usp.br \\ and A. DE A. E SILVA \\ Departamento de Matemática, Universidade Federal da Paraíba, CEP 58051-900, João Pessoa, Pb, Brazil \\ e-mail: andrade@mat.ufpb.br
}

(Received 30 June 2010; accepted 23 November 2011)

\begin{abstract}
Let $G$ be a group such that, for any subgroup $H$ of $G$, every automorphism of $H$ can be extended to an automorphism of $G$. Such a group $G$ is said to be of injective type. The finite abelian groups of injective type are precisely the quasi-injective groups. We prove that a finite non-abelian group $G$ of injective type has even order. If, furthermore, $G$ is also quasi-injective, then we prove that $G=K \times B$, with $B$ a quasi-injective abelian group of odd order and either $K=Q_{8}$ (the quaternion group of order 8) or $K=\operatorname{Dih}(A)$, a dihedral group on a quasi-injective abelian group $A$ of odd order coprime with the order of $B$. We give a description of the supersoluble finite groups of injective type whose Sylow 2-subgroup are abelian showing that these groups are, in general, not quasi-injective. In particular, the characterisation of such groups is reduced to that of finite 2-groups that are of injective type. We give several restrictions on the latter. We also show that the alternating group $A_{5}$ is of injective type but that the binary icosahedral group $\operatorname{SL}(2,5)$ is not.
\end{abstract}

2000 Mathematics Subject Classification. Primary 20D45, Secondary 20 F28.

1. Introduction. Eilenberg and Moore's theorem states that the category of finite groups does not contain non-trivial injective objects (see [3] and [6]). To sidestep this, the following weaker condition, called quasi-injectivity, was introduced. A group $G$ is said to be quasi-injective if for every subgroup $H$ of $G$ and every homomorphism $\phi: H \rightarrow G$ there exists an endomorphism $\psi: G \rightarrow G$ that extends $\phi$. In [2], it is remarked that quasi-injective finite groups are precisely those finite groups $G$ for which all endomorphism of subgroups can be extended to endomorphisms of G. Quasiinjective abelian groups were classified by L. Fuchs [4]: These are either divisible abelian 
groups or are periodic and each Sylow $p$-subgroup is a direct product of isomorphic cyclic or quasi-cyclic groups (i.e. homocyclic groups).

Bertholf and Walls classified finite quasi-injective groups $G([2])$ and Tomkinson in [9] handled the soluble and locally finite case. In particular, it is proved that a locally finite quasi-injective group is locally supersoluble, metabelian and a $T$-group. The latter means that every subnormal subgroup is normal. In general, the classification of quasi-injective groups is still not settled.

In [1], these investigations were continued focusing on extensions of automorphism of subgroups. We recall the precise definition given to this. A group $G$ is said to be of injective type if for every subgroup $H$ of $G$ and every $\phi \in \operatorname{Aut}(\mathrm{H})$ there exists $\psi \in \operatorname{Aut}(\mathrm{G})$ such that $\phi=\psi_{\mid H}$. So, a necessary condition for a finite group $G$ to be of injective type is that $|\operatorname{Aut}(H)|$ divides $|\operatorname{Aut}(G)|$, for every subgroup $H$ of $G$. Clearly, the group $\mathbb{Z}$ is an abelian group of injective that, by the result of Fuchs, is not quasiinjective. In [1], the abelian groups of injective type have been described. It follows that a finite abelian group is of injective type if and only if it is quasi-injective. The nonperiodic abelian groups $A$ of injective type are those with a divisible periodic subgroup $T(A)$ so that $A / T(A)$ has rank one. Furthermore, it is shown that a finite nilpotent group of odd order which is of injective type must be abelian.

In Section 2, we classify among the finite groups of injective type those that are also quasi-injective. Next we show that finite groups of injective type must have even order and classify finite supersoluble groups of injective type with abelian Sylow 2subgroups. In Section 3, we show that (non-abelian) Sylow 2-subgroups of a finite group $G$ of injective type can neither be a dihedral, nor a quaternion group of order strictly larger than 8. However, if $G$ is a non-abelian finite group of injective type, with a non-central element of order at least three, then $\operatorname{Aut}(G)$ has a section that is a dihedral group. It follows that $|\operatorname{Aut}(G)|$ is even and hence $|\operatorname{Aut}(G)|$ is even for all groups of injective type (excluding $C_{2}$ ). In the last section, we show that the alternating group $A_{5}$ is of injective type but the binary icosahedral group $\operatorname{SL}(2,5)$ is not. On the other hand, notice that a quasi-injective group is always soluble (see [2] and [9]).

The notation used is mostly standard and follows that of [5] and [8]. By $G_{p}$ we denote an element of $\operatorname{Syl} l_{p}(G)$, the set of Sylow $p$-subgroups of the finite group $G$, and by $\phi_{g}$ we denote conjugation by $g \in G$ given by the mapping $x \mapsto g^{-1} x g$.

2. Groups of injective type. We begin with recalling three elementary properties stated in [1].

LEMMA 2.1. Let $G$ be a group of injective type and $H$ a characteristic subgroup of $G$. Then $H$ is of injective type.

LEMMA 2.2. Let $G$ be a group of injective type, $H$ a subgroup of $G, \phi \in \operatorname{Aut}(\mathrm{H})$ and $\psi \in \operatorname{Aut}(\mathrm{G})$ such that $\phi=\psi_{\mid H}$. Then

(1) $\psi\left(\mathcal{C}_{G}(H)\right)=\mathcal{C}_{G}(H)$, the centraliser of $H$ in $G$.

(2) $\psi\left(\mathcal{N}_{G}(H)\right)=\mathcal{N}_{G}(H)$, the normaliser of $H$ in $G$.

Lemma 2.3. Let $G=N \rtimes X$, a semidirect product of the groups $N$ and $X$. If $G$ is of injective type and $N$ is characteristic in $G$ then $X$ is of injective type.

Another useful and easily proved property is the following. 
Lemma 2.4. Suppose $G=N \times X$, the direct product of two groups $N$ and $X$. If $(|N|,|X|)=1$ then $G$ is of injective type if and only if $N$ and $X$ are of injective type.

Let $p$ be a prime and $r \in \mathbb{N}$. Recall that a $p$-group $G$ is said to be homocyclic of type $\left(p^{r}, m\right)$ if it is the direct product of $m$ copies of the cyclic group of order $p^{r}$. Here, $m$ is allowed to be any cardinal. We set $\Omega_{p^{k}}(G)=\left\langle g \in G: g^{p^{k}}=1\right\rangle$.

Also recall that an automorphism of a group $G$ is said to be a power automorphism if it leaves invariant every subgroup of $G$. The subgroup generated by the power automorphisms is denoted by $\operatorname{Paut}(G)$.

If $A$ is an abelian $p$-group of finite exponent and $\phi \in \operatorname{Paut}(A)$, then there exists $l \in \mathbb{Z}$ such that $\phi(a)=a^{l}$, for all $a \in A$ (see $\left.[8,13.4 .3]\right)$. Hence, in this case, $\operatorname{Paut}(A) \subseteq$ $\mathcal{Z}(\operatorname{Aut}(A))$. Moreover, if $\phi$ is non-trivial and has order prime to $p$, then it is also fixed-point free.

If $\sigma$ is a $p^{\prime}$-automorphism of the abelian $p$-group $A$. Then (see [5, Chapter 5, 2.4]) $\sigma$ acts trivially on $A$ if and only if it acts trivially on $\Omega_{p}(A)$. More generally, if $\sigma$ is a $p^{\prime}$-automorphism of the $p$-group $A$, with $p$ odd, then (see [5, Chapter 5, 3.10]) $\sigma$ acts trivially on $A$ if and only if it acts trivially on $\Omega_{p}(A)$. Therefore, an involution which is in $\operatorname{Paut}(A)$ and acts non-trivial on $\Omega_{p}(A)$ must be inversion and hence, in this case, $\operatorname{Paut}(A)$ has a unique involution.

Since we need the classification of finite quasi-injective groups, we recall the result of Bertholf and Walls. It shows how $\operatorname{Paut}(G)$ comes naturally into the picture when studying quasi-injective groups. We denote by $Q_{8}$ the quaternion group of order 8 .

THEOREM 2.5 (Bertholf-Walls [2]). A finite group $G$ is quasi-injective if and only if either $G=Q_{8} \times A$, with $A$ a quasi-injective finite abelian group of odd order or $G=$ $K \rtimes H$ such that

(1) $K_{p}$ and $H_{p}$ are homocyclic for each prime $p$;

(2) $G^{\prime}=K$;

(3) $(|K|,|H|)=1$;

(4) For each $h \in H$, if $p$ is a prime divisor of $|K|$ then $\phi_{h} \in \operatorname{Paut}\left(K_{p}\right)$;

(5) If $K_{\pi}$ is a Hall $\pi$-subgroup of $K$ (for some set of primes $\pi$ ) then $\mathcal{C}_{H}\left(K_{\pi}\right)$ is a direct factor of $H$. In particular, $\mathcal{Z}(G) \cap H=\mathcal{C}_{H}(K)$ is a direct factor of $G$.

For an abelian group $A$ we put $\operatorname{Dih}(A)=A \rtimes C_{2}$, the semidirect product, where the cyclic group $C_{2}$ of order two acts by inversion on $A$.

THEOREM 2.6. Let $G$ be a finite non-abelian group that is quasi-injective. Then, $G$ is of injective type if and only if $G \cong K \times B$, with $B$ a quasi-injective abelian group of odd order and either $K=Q_{8}$ or $K \cong \operatorname{Dih}(A)$ with $A$ a quasi-injective abelian group of odd order coprime with the order of $B$.

Proof. Let $G$ be a finite non-abelian group of injective type. First assume $G$ is quasi-injective. So, $G$ is as described in Theorem 2.5. Because of Lemma 2.4, to prove the necessity of the conditions of the mentioned conditions, it is sufficient to deal with the case that $G=K \rtimes H$, with $K$ and $H$ as in Theorem 2.5. So, if $p$ is a prime divisor of $|K|$ and $h \in H$, then $\phi_{h}$ induces a power automorphisms on $K_{p}$. Denote by $\psi$ an extension of the inversion $\phi$, say, on $\langle h\rangle$. Using the fact that the restriction of $\phi_{h}$ on $K$ is central in $\operatorname{Aut}(K)$, we obtain that $\phi_{h}=\psi \phi_{h} \psi^{-1}=\phi_{\psi(h)}=\phi_{h^{-1}}$ and hence $\phi_{h^{2}}=1$ (all homomorphisms are considered restricted to $K$ ). It follows that $H^{2}$ centralises $K$. Hence, if $|G|$ were odd, then $H$ would centralise $K$ and so $G$ would be abelian, a contradiction. So $|H|$ must be even, $A=O_{2^{\prime}}(H)$ acts trivially on $K$ and so 
is central in $G$. Because $G$ is, by assumption, non-abelian, there exists a non-trivial element of $H / H^{2} \simeq H_{2} / H_{2}^{2}$, say $\bar{a}$, that acts non-trivially on $K$. Furthermore, as $H_{2}$ is homocyclic, for any other non-trivial element $\bar{b}$ of $H / H^{2}$ there exits an automorphism of $H$ that induces an automorphism of $H / H^{2}$ mapping $\bar{a}$ to $\bar{b}$. Because $G$ is of injective type, such an automorphism is the restriction of an automorphism of $G$. As $a$ is not central in $G$ it follows that $b$ is not central in $G$. Because of Item 4 in Theorem 2.5, it thus follows that the elementary abelian 2-group $H / H^{2} \simeq H_{2} / H_{2}^{2}$ is isomorphic with a subgroup of $\operatorname{Paut}\left(K_{p}\right)$. Hence, as remarked before Theorem 2.5, $H / H^{2}$ must be isomorphic to $C_{2}$. Since $H_{2}$ is homocyclic, we thus get that $H \simeq C_{2^{r}} \times O_{2^{\prime}}$. Again using that $H^{2}$ is central in $G$, we obtain from Item 5 of Theorem 2.5 that $r=1$ and thus $H \simeq C_{2} \times O_{2^{\prime}}$. So, $G \simeq\left(K \rtimes C_{2}\right) \times H_{2^{\prime}}$. As $C_{2}$ acts non-trivial on each non-trivial $K_{p}$, again by the comment before Theorem 2.5, the action of $C_{2}$ on $K$ is inversion. Hence, $G=\operatorname{Dih}(K) \times H_{2^{\prime}}$, as desired.

Because of Lemma 2.4 and Theorem 2.5, to prove the converse it is sufficient to prove that if $G=\operatorname{Dih}(A)$, with $A$ a homocyclic group of odd order, then $G$ is of injective type. Let $H$ be a subgroup of $\operatorname{Dih}(A)$. If $H \subseteq A$, then, by the results mentioned in the introduction, any automorphism of $H$ extends to $A$ and we get an extension to all of $G$ defining it as the identity on any element of order 2 . So, we may suppose that $H=\operatorname{Dih}(C)$ with $C \subseteq A$. Since $C$ has odd order, it is characteristic in $H$ and thus, any automorphism of $H$ restricts to an automorphism of $C$. Once again we can extend any automorphism $\phi$ of $H$ to all of $G$ by first extending $\phi_{\mid C}$ to an automorphism of $A$ and then extending this automorphism to $G$ imposing that on some 2-element of $H$ it acts as $\phi$.

Together with the classification theorem of Bertholf and Walls, this theorem shows that the class of finite groups that are quasi-injective differs from the class of finite groups of injective type. We give an easy example. Let $C_{7}=\left\langle x \mid x^{7}=1\right\rangle, C_{3}=\langle y|$ $\left.y^{3}=1\right\rangle$ and $G=C_{7} \rtimes C_{3}$, with $x^{y}=x^{2}$. Then $G$ is quasi-injective but is not of injective type. This example also shows that if $G$ has a normal subgroup $H$ such that $H$ and $G / H$ are of injective type, then $G$ need not be of injective type. On the other hand, a typical group which is both quasi-injective and of injective type is $\operatorname{Dih}\left(C_{p^{r}}\right)$, with $p$ an odd prime. Note that the indecomposable groups of Theorem 2.6, different from $Q_{8}$, are all Frobenius groups.

As mentioned in the introduction, Tomkinson extended the result of Bertholf and Walls and showed that a locally finite quasi-injective group is metabelian and locally soluble.

THEOREM 2.7. Let $G$ be a finite non-abelian group of injective type. Then $|G|$ is even.

Proof. Let $G$ be a counter example of minimal order. By Feith-Thompson's Theorem, $G$ is soluble. Denote the last non-trivial term of the lower central series of $G$ by $L$. Because $L$ is characteristic in $G$ we get from Lemma 2.1 that $L$ is of injective type. As, by assumption, $G$ is a counter example of minimal order, it follows that $L$ is abelian. Hence (see for example [8,9.2.7]), we have that $G=L \rtimes N$, where $N$ is a system normaliser and thus a nilpotent group. Because of Lemma 2.3, we thus also get that $N$ is a finite nilpotent group of injective type. Consequently, as mentioned in the introduction, the subgroup $N$ is abelian. Therefore, $G^{\prime} \subseteq L$ and thus $L=G^{\prime}$.

We claim that $(|L|,|N|)=1$. Suppose the contrary and let $p$ be a common prime divisor of $|L|$ and $|N|$. So, $N_{p}$ and $L_{p}$ are non-trivial and $S_{p}=\left\langle L_{p}, N_{p}\right\rangle$ is a Sylow $p$-subgroup of $G$. Choose $1 \neq g_{0} \in L_{p} \cap \mathcal{Z}\left(S_{p}\right)$ of order $p$ and $g_{1} \in N_{p}$ also of order 
$p$. Then, since $L \bigcap N=1$ and $G$ is of injective type, there exist $\psi \in \operatorname{Aut}(G)$ such that $\psi\left(g_{0}\right)=g_{1}$. But, since $L_{p}$ is characteristic in $G$, it follows that $g_{1} \in L_{p}$, a contradiction. This proves the claim.

Next we claim that $L$ is a $p$-group for some prime $p$. If not, then $L=L_{p} \times L_{p^{\prime}}$ for some non-trivial $p^{\prime}$-subgroup $L_{p^{\prime}}$ of $L$. As $L_{p}$ and $L_{p^{\prime}}$ are characteristic subgroups of $G$, we get that $\left\langle L_{p}, N\right\rangle=L_{p} \rtimes N$ and $\left\langle L_{p^{\prime}}, N\right\rangle=L_{p^{\prime}} \rtimes N$ are proper subgroups of $G=$ $L_{p^{\prime}} \rtimes\left(L_{p} \rtimes N\right)=L_{p} \rtimes\left(L_{p^{\prime}} \rtimes N\right)$. From Lemma 2.3 and the minimality assumption, we thus obtain that $L_{p} \rtimes N$ and $L_{p^{\prime}}$ are abelian. Hence, $G$ is abelian, a contradiction. Hence, indeed $L$ is an abelian $p$-group.

A similar argument also shows that $N$ is of prime power order. Indeed for otherwise, write $N=N_{q_{1}} \times N_{q_{2}}$ (with $q_{1}$ and $q_{2}$ positive integers that are coprime) and consider the proper characteristic subgroups $L \rtimes N_{q_{1}}$ and $L \rtimes N_{q_{2}}$. Because of Lemma 2.1, both subgroups are of injective type. The minimality assumption implies that these are abelian and thus $G$ is abelian, a contradiction.

So we proved that $G=L \rtimes N$, with both $L$ and $N$ abelian groups of prime power order.

Now let $x \in N$. Assume there exists $y \in L$ such that $y^{x} \notin\langle y\rangle$. Since $\langle y\rangle \neq\left\langle y^{x}\right\rangle$ and $G$ is of injective type, there exists $\psi \in \operatorname{Aut}(G)$ such that $\psi(y)=y^{-1}$ and $\psi\left(y^{x}\right)=y^{x}$. But then $y^{x}=\psi\left(y^{x}\right)=\left(y^{-1}\right)^{\psi(x)}$ and hence $x^{-1} \psi(x)$ inverts $y$. Since, $y$ is of odd order, we obtain that $x^{-1} \psi(x)$ is of even order, a contradiction. It follows that $x$ induces a power automorphism of $L$ and so, as mentioned earlier, there exists $l \in \mathbb{Z}$ such that $y^{x}=y^{l}$, for all $y \in L$.

We are now in a position to finish the proof. Since $G$ is non-abelian, there exists $y \in L$ and $x \in N$ such that $y^{x} \neq y$. Hence, by the previous, $\phi_{x} \in \operatorname{Paut}(L)$ and there exists $k \in \mathbb{N}$ such that $z^{x}=z^{k}$, for $z \in L$. Because $x$ has odd order and $G$ is of injective type, there exists $\Psi \in \operatorname{Aut}(G)$ such that $\Psi(x)=x^{2}$. Let $z=\Psi(y)$. It follows that

$$
\Psi(y)^{k^{2}}=z^{k^{2}}=z^{x^{2}}=\Psi(y)^{x^{2}}=\Psi(y)^{\Psi(x)}=\Psi\left(y^{x}\right)=\Psi\left(y^{k}\right)=\Psi(y)^{k} .
$$

Hence, $o(y)$ divides $k^{2}-k$. Because $k$ and $o(y)$ are coprime, we conclude that $o(y)$ divides $k-1$. Consequently, $y^{x}=y$, a contradiction. Hence, $G$ indeed is of even order.

Our next result gives a characterisation of supersoluble finite groups that are of injective type and whose Sylow 2-subgroup are abelian.

THEOREM 2.8. Let $G$ be a finite supersoluble finite group with $G_{2}$ abelian. Then, $G$ is of injective type if and only if either $G$ is abelian or $G \simeq A \times(K \rtimes H)$, where

(1) $A$ and $K$ are abelian groups of odd order and of injective type,

(2) $(|A|,|K|)=1$,

(3) $H \cong C_{2^{n}}$, for some integer $n \geq 1$.

(4) $k^{t}=k^{-1}$, for all $k \in K$ and $t \in H \backslash \mathcal{C}_{H}(K)$.

Proof. Suppose that $G$ is of injective type. Since $G$ is finite and supersoluble it follows, by $[\mathbf{8}, 5.4 .9]$, that $W=\{g \in G: o(g)$ odd $\}=\mathcal{O}_{2^{\prime}}(G)$. Therefore, $W$ is of injective type (Lemma 2.1), has odd order and hence, by the previous theorem, is abelian and (by Theorem 2.5) its Sylow subgroups are homocyclic characteristic subgroups of $G$. Schur-Zassehaus's theorem ([8,9.1.2]) guarantees that $G=W \rtimes H$ with $H \in S_{y l} l_{2}(G)$. By hypothesis, $H$ is an abelian and, since it is also of injective type, it is homocyclic. If $H$ acts trivially on $W$ then $G$ is abelian and so we may suppose that it acts non-trivial 
on $W$. Setting $A=\mathcal{C}_{W}(H)$, we will show that $A$ is a direct factor of $G$. To prove this, we shall prove that $H=\left\langle h_{0}\right\rangle$ and that $h_{0}$ either centralises or acts as inversion on the Sylow subgroups of $W$. Hence, we may write $W=A \times K$, with $h_{0}$ acting as inversion on $K$.

To prove the last assertions, let $p$ be a prime divisor of $|W|$ and suppose that $H$ acts non-trivially on $W_{p}$. Write $G=O_{p^{\prime}}(G) \rtimes\left(W_{p} \rtimes H\right)$. As $O_{p^{\prime}}(G)$ is characteristic in $G$, it follows from Lemma 2.4 that the subgroup $V=W_{p} \rtimes H$ is of injective type and is supersoluble.

Since $p>2$, Zappa's result $([8,5.4 .8])$ implies that there exists $x \in W_{p}, o(x)=p$, such that $L=\langle x\rangle$ is normal in $V$. Suppose there exists $h \in H$ such that $h^{2} \notin \mathcal{C}_{H}(L)$ but $h^{4} \in \mathcal{C}_{H}(L)$. Then $(L \rtimes\langle h\rangle) /\left\langle h^{4}\right\rangle \simeq L \rtimes C_{4}=L \rtimes\langle\bar{h}\rangle$, where the action defining the semidirect product is given by $x^{\bar{h}}=x^{h}=x^{k}$, and $\operatorname{gcd}(k, p)=1$. Let $N=\langle h\rangle$ and let $\phi \in \operatorname{Aut}(N)$ be inversion. Then, since $G$ is of injective type, there exists $\psi \in \operatorname{Aut}(G)$ such that $\left.\psi\right|_{N}=\phi$. Thus,

$$
\psi(x)^{k}=\psi\left(x^{\bar{h}}\right)=\psi\left(x^{h}\right)=\psi(x)^{h^{-1}} \text { and } \overline{\psi(x)}^{k}=\overline{\psi(x)}^{\bar{h}^{-1}}=\overline{\psi(x)}^{\bar{h}^{3}}=\overline{\psi(x)}^{k^{3}} .
$$

Hence, $p \mid\left(k^{3}-k\right)$, i.e., $p \mid\left(k^{2}-1\right)$, because $\operatorname{gcd}(k, p)=1$. It follows that $\left[h^{2}, x\right]=1$, a contradiction. So we proved that $h^{2} \in \mathcal{C}_{H}(L)$ for all $h \in H$.

Now, let $z \in \Omega_{p}\left(W_{p}\right)$ and $h \in H$. Suppose $z \notin\langle x\rangle$. Then, since $V$ is of injective type, there exists $\Psi \in \operatorname{Aut}(V)$ such that $\Psi(x)=z$. From this we get that $z=\Psi\left(x^{\Psi^{-1}\left(h^{2}\right)}\right)=$ $z^{h^{2}}$, for all $h \in H$. It follows that $h^{2} \in \mathcal{C}_{H}\left(W_{p}\right)$ and, consequently, $h^{2} \in \mathcal{C}_{H}\left(W_{p}\right)$ (see the comments after Lemma 2.4). Since $\langle x\rangle \triangleleft V$ we also have that $z^{h}=\Psi\left(x^{\Psi^{-1}(h)}\right) \in$ $\langle z\rangle$. This shows that any $h \in H$ acts as a power automorphism on $\Omega_{p}\left(W_{p}\right)$ and, as $h^{2}$ centralises $\Omega_{p}\left(W_{p}\right)$, this action is an involution. Thus, invoking once more the comments after Lemma 2.4, we have that each $h \in H$ either centralises or acts as inversion on $\Omega_{p}\left(W_{p}\right)$. Since $p$ is odd we have that $h$ either centralises or acts as inversion on $W_{p}$.

The former, and the structure of $V$, show that $\mathcal{C}_{H}\left(W_{p}\right)$ is central in $H$, characteristic and its index in $H$ is 2 . Hence $\mathcal{C}_{H}\left(W_{p}\right)$ and $H$ are homocyclic and $\left[H: \mathcal{C}_{H}\left(W_{p}\right)\right]=2$. If $\mathcal{C}_{H}\left(W_{p}\right) \neq 1$ then, since it is characteristic in $H$ and $H$ is of injective type, it contains $\Omega_{2}(H)$. Consequently, as both are homocyclic groups, they must have the same rank. It follow that $H \cong C_{2^{n}}$, for some integer $n \geq 2$. Obviously, if $\mathcal{C}_{H}\left(W_{p}\right)=1$ then $H \cong C_{2}$.

Conversely, suppose that $G$ is as described, $V$ is a subgroup of $G$ and $\phi \in \operatorname{Aut}(V)$. We have to prove that $\phi$ can be lifted to an automorphism of $G$. Write $V=V_{A} \times$ $\left(V_{K} \rtimes V_{H}\right.$ ), where the subscripts have the obvious meaning (for example $V_{K}=V \cap$ $K)$. Clearly, $\phi$ fixes $V_{A}$ and $V_{K}$. The Schur-Zassenhaus theorem guarantees that the complements of the kernels of the semidirect products involved all are conjugated to each other. Hence, if necessary, conjugating by an element of $G$ we also may assume that $\phi$ fixes $V_{H}$. Because of the assumptions, we have that $k^{h}=k^{-1}$, for any $h \in H \backslash \mathcal{C}_{H}(K)$ and all $k \in K$. Denote by $\phi_{A}, \phi_{K}$ and $\phi_{H}$ the restrictions of $\phi$ to $V_{A}, V_{K}$ and $V_{H}$, respectively. Extend each one of them to $A, K$ and $H$, respectively and denote these extensions by $\Psi_{A}, \Psi_{K}$ and $\Psi_{H}$ (such extension exists because of the assumptions 1 . and 3.). Then it is readily verified that the mapping $\Psi: G \rightarrow G$ defined by mapping akh to $\Psi_{A}(a) \Psi_{K}(k) \Psi_{H}(h)$ (with $a \in A, k \in K$ and $h \in H$ ) yields an automorphism of $G$ that is an extension of $\psi$, as desired.

It remains an open problem to characterise the finite 2-groups that are of injective type. In the next section, we will prove some information on their structure. 
We finish this section by giving an example of a finite group of injective type that is not quasi-injective. Let $G \simeq K \rtimes C_{2^{n}}$, with $n \geq 2, C_{2^{n}}=\left\langle x \mid x^{2^{n}}=1\right\rangle, K$ a homocyclic $p$-group, $p$ an odd prime and $x$ acts via inversion on $K$. Then, by Theorem $2.8, G$ is of injective type. But, because of Theorem 2.5 and since $\mathcal{Z}(G)=C_{2^{n-1}}$ is not a direct factor of $C_{2^{n}}$, the group $G$ is not quasi-injective.

3. Obstruction to injectiveness. Here, we give some obstruction for a finite group to be of injective type. By $D_{2^{m}}$ (respectively $Q_{2^{m}}$ ), we denote the dihedral (respectively quaternion) group of order $m$.

Proposition 3.1. Let $G$ be a finite group of injective type.

(1) Let $p$ be a prime divisor of $|\mathcal{Z}(G)|$. Then there exists a positive integer $n$ such that $\operatorname{Syl}_{p}(\mathcal{Z}(G))=\Omega_{p^{n}}(G)$. Consequently, if, moreover, $G$ is a 2-group, then $\mathcal{Z}(G)=$ $\Omega_{2^{n}}(G)$, with $2^{n}$ the exponent of $\mathcal{Z}(G)$ and thus $D_{8}$ is not a subgroup of $G$.

(2) Let $A$ be a characteristic subgroup of $G$ and $p$ a prime divisor of $|A \cap \mathcal{Z}(G)|$. Then $\Omega_{p}(G) \subseteq A$.

(3) If $Q_{8}$ is a subgroup of $G$, then either all cyclic subgroups of order 4 of $Q_{8}$ are normal in $G$ or none of them are. Consequently, $Q_{2^{n}}$ is not a characteristic subgroup of $G$.

Proof. Suppose $a$ and $b$ are elements of $G$, with $|\langle a\rangle|=|\langle b\rangle|=p^{k}$, such that $a \in$ $\mathcal{Z}(G)$ and $\langle a\rangle \cap\langle b\rangle=1$. Then $H=\langle a, b\rangle=\langle a\rangle \times\langle b\rangle$ is a subgroup of $G$. Let $\phi \in$ $\operatorname{Aut}(H)$ defined by $\phi(a)=b$ and $\phi(b)=a$. Then there exists $\psi \in \operatorname{Aut}(G)$ such that $\left.\psi\right|_{H}=\phi$. It follows that $b=\psi(a) \in \mathcal{Z}(G)$.

Let now $g \in G$ be an element of order $p^{k}$, where $p^{k}$ is at most the exponent of $\operatorname{Syl}_{p}(\mathcal{Z}(G))$, and let $a \in \mathcal{Z}(G)$ be an element of order $p^{k}$. Then, by the previous, $\langle g\rangle \cap\langle a\rangle \neq\{1\}$. Hence, $W=\langle g, a\rangle \cong C_{p^{k}} \times C_{p^{l}}$, with $0<l<k$, and $\langle g\rangle$ and $\langle a\rangle$ are direct summands of $W$. Therefore, there exists $\phi \in \operatorname{Aut}(W)$ so that $\phi(a)=g$. As $G$ is of injective type, there exists a $\psi \in \operatorname{Aut}(G)$ so that $\psi_{\mid W}=\phi$. Consequently, $g=\psi(a)$ is a central element of $G$. It follows that $S y l_{p}(\mathcal{Z}(G))=\Omega_{p^{n}}(G)$ for some $n$.

Assume now that $G$ also is a 2-group. Since elements of order 2 in $D_{8}$ are central in $D_{8}$, it follows from the previous that $D_{8}$ cannot be a subgroup of $G$. Hence, the first part of the statement follows.

The proof of the second item is basically the same as that of the first item.

To prove the third part. Let $Q_{8}=\langle a, b\rangle$. Then there exists an automorphism of $Q_{8}$ so that $\phi(a)=b$. Because $G$ is of injective type, there exists an automorphism $\Psi$ of $G$ so that $\Psi_{\left.\right|_{Q_{8}}}=\phi$. So, if $\langle a\rangle$ is normal in $G$, then so is $\langle b\rangle$. Hence, the result follows.

Note that it follows from the second part of the proposition that if $G=Q_{8} \times A$ is of injective type, with $A$ a finite 2-group, then $A$ is trivial. Indeed, if $A$ has an element, say $c$ of order 4 and $Q_{8}=\langle a, b\rangle$ then $H=\langle a\rangle$ and $K=\langle a c\rangle$ are subgroups of order 4 and $L=H K=H \times K$. Let $\phi \in \operatorname{Aut}(L)$ be such that $\phi(a)=a c$. Thus, if $G$ were of injective type, then there exists $\psi \in \operatorname{Aut}(G)$ such that $\psi_{\mid H}=\phi$. Since $H$ is a normal subgroup in $G$ we have that $K=\psi(H)$ is a normal subgroup in $G$, a contradiction, because $(a c)^{b}=a^{-1} c=a^{2}(a c) \notin K$. On the other hand, if $A$ were elementary abelian then choose $\langle c\rangle \subset A$. Note that $c$ is not a square in $G$. Set $H=\left\langle a^{2}\right\rangle$ and $K=\langle a c\rangle$. If $G$ were of injective type then we can find an automorphism $\Psi$ of $G$ such that $c=\Psi\left(a^{2}\right)=\Psi(a)^{2}$ and hence $c$ would be a square, a contradiction. 
The previous also shows that if $G$ is a finite 2-group of injective type, and $y \in G$ is such that $y^{2} \in \mathcal{Z}(G)$ has order 2 then any element of $\mathcal{Z}(G)$ that is of order 2 is a square of some element of $G$.

REMARK 3.2. Let $G$ be a finite group of injective type and $y \in G$ a non-central element, $o(y) \geq 3$. Then there exists $\psi \in \operatorname{Aut}(G)$, whose order is a power of 2 , such that $\psi(y)=y^{-1}$. It follows that $\psi \phi_{y} \psi^{-1}=\phi_{y^{-1}}$. Consequently, $\operatorname{Aut}(G)$ has a section which is a dihedral group and hence $|\operatorname{Aut}(G)|$ is even. It is now easily seen that if $G$ is of injective type then $|\operatorname{Aut}(G)|$ is either trivial or has even order.

Lemma 3.3. Let $G$ be a a finite group and $P$ a Sylow 2-subgroup of $G$. If $P$ is isomorphic to $D_{2^{n}}, n \geq 3$ or $Q_{2^{n}}, n \geq 4$, then $G$ is not of injective type.

Proof. The main idea is the same in both cases. Let $G$ be any of the two groups and let $\langle a\rangle$ be the maximal cyclic characteristic subgroup of $P$. Choose $b \notin\langle a\rangle$ such that $o(b)=o\left(a^{2^{k}}\right)$ and set $c=a^{2^{k}}, H=\langle b, c\rangle$. In any case, there exist $\phi \in \operatorname{Aut}(H)$ such that $\phi(b)=c$ and $\phi(c)=b$. If $G$ were of injective type then $\phi$ would be the restriction of $\psi \in \operatorname{Aut}(G)$. It follows that $\langle\psi(a)\rangle$ is a characteristic subgroup of $\psi(P)$ and $b$ would be in this characteristic subgroup. Hence, $b$ would be a square in $P$, a contradiction.

4. Non-soluble examples. We finish with looking at two finite non-soluble groups one of which is of injective type but the other fails to be. In the latter case, we exhibit the subgroup having an automorphisms, which does not extend to an automorphism of the whole group.

EXAMPLE 4.1. The alternating group $A_{5}$ is of injective type. More precisely, if $H<A_{5}$ and $\phi \in \operatorname{Aut}(H)$ then there exists $g \in S_{5}$ such that $\phi=\left(\phi_{g}\right)_{\left.\right|_{H}}$.

Proof. Let $H$ be a subgroup of $A_{5}$. We need to show that any automorphism $\Psi$ of $H$ can be extended to an automorphism of $A_{5}$. Actually, we will show that each $\Psi$ can be lifted to an automorphism that is induced by conjugation with an element of $S_{5}$. This is clear in case $H$ is cyclic, as elements of the same cyclic structure in $S_{n}$ are conjugate. If $H$ has order 6 then $H \cong S_{3}$ and so all its automorphisms are inner and hence the claim follows. If $H$ has order 10 , then $H$ is conjugate to $M=\langle(12345)\rangle \rtimes\langle(14)(23)\rangle$ and we have that $\operatorname{Out}(M)$ has order 2 and is generated by the element which sends (12345) to its square and fixes (14)(23). But this automorphism is induced by conjugation with $(1243) \in S_{5}$. So, again the claim follows. Assume now that $H$ has order 4 . Then $H$ is isomorphic to the Klein four-group and all such groups are conjugated in $A_{5}$ (as they are 2-Sylow subgroups). Without the loss of generality, we may assume that $H \subset A_{4} \subset A_{5}$ and we know that $H \triangleleft S_{4} \subset S_{5}$. Since the elements of order 2 of $A_{4}$ are conjugate in in $S_{5}$, it follows that the normaliser of $H$ in $S_{5}$ contains a subgroup isomorphic to $S_{3}$ which in turn is isomorphic to $\operatorname{Aut}(H)$ and thus the claim follows. Finally, it remains to deal with $H$ of order 12 . In this case, $H \cong A_{4}$ and is the set of even permutations of one of the following sets $\{1,2,3,4\},\{1,2,3,5\},\{1,2,4,5\},\{1,3,4,5\},\{2,3,4,5\}$. Hence, since $\operatorname{Aut}\left(A_{4}\right) \cong S_{4} \cong \operatorname{Inn}\left(S_{4}\right)$ and $N_{S_{5}}\left(A_{4}\right) \cong S_{4} \subset S_{5}$, the claim again follows.

The group $G=S L(2,5)$ is not of injective type. Nearly all its subgroups have the property that their isomorphisms can be lifted to isomorphisms of $G$. Only the subgroups of order 20 do not have this property. We show how this works since it 
might be useful. In fact, [7, Theorem 18.6] guarantees the existence of a Frobenius complement $G_{0}$, say, of order 240 such that $G_{0} / \mathcal{Z}\left(G_{0}\right) \cong S_{5}$, the symmetric group on five elements, and contains $S L(2,5)$ as a subgroup of index 2 . We also have $S L(2,5) / \mathcal{Z}\left(G_{0}\right) \cong A_{5}$ and that $\mathcal{Z}\left(G_{0}\right)$ is the unique subgroup of order 2 of $G_{0}$. Let $H<G$ and $H_{0}=H \cap \mathcal{Z}\left(G_{0}\right)$. Denote by $\bar{H}$ the image of $H$ in $A_{5}<S_{5}$ and let $\phi \in \operatorname{Aut}(H)$; then $\phi\left(H_{0}\right)=H_{0}$ and so $\phi$ induces an isomorphism $\bar{\phi} \in \operatorname{Aut}(\bar{H})$, given by $\bar{\phi}(\bar{g})=\overline{\phi(g)}$. By Example 4.1, it follows that $\bar{\phi}=\phi_{\bar{x}}$, for some $x \in G_{0}$. So we have that $\phi(h)=x^{-1} h x$ or $\phi(h)=x^{-1} h x z_{0}$, where $\mathcal{Z}\left(G_{0}\right)=\left\langle z_{0}\right\rangle$. Hence, if $o(h)$ is odd then $\phi(h)=x^{-1} h x . H$ is one of the following groups: $C_{2}, C_{3}, C_{4}, C_{5}, C_{6}, K_{8}, C_{10}, C_{3} \rtimes C_{4}$ with a generator of $C_{4}$ inverting $C_{3}, C_{5} \rtimes C_{4}$ with a generator of $C_{4}$ inverting $C_{5}$ or $K_{8} \rtimes C_{3}=S L(2,3)$. If $H$ is cyclic then $\phi$ can obviously be lifted to $G$. In case $H=K_{8}$, note that $N_{G_{0}}(H)$ is the binary octahedral group of order 48 and $\mathcal{C}_{G_{0}}(H)=\mathcal{Z}\left(G_{0}\right)$. So we have that $N_{G_{0}}(H) / \mathcal{C}_{G_{0}}(H) \cong S_{4}=\operatorname{Aut}\left(K_{8}\right)$ and hence once more $\phi$ is given by conjugation with an element of $G_{0}$. If $H=S L(2,3)$, then it is generated by its elements of order 3. For an element $h$ of order 3 , we have that $\phi(h)=x^{-1} h x$ and hence this holds for all elements of $H$. Hence, we are left with $H \in\left\{C_{3} \rtimes C_{4}, C_{5} \rtimes C_{4}\right\}$.

In case $H=C_{3} \rtimes C_{4}=\langle a\rangle \rtimes\langle b\rangle$ we have that $\operatorname{Aut}(H) \cong\left\langle\phi_{1}\right\rangle \times\left(\left\langle\phi_{a}\right\rangle \rtimes\left\langle\phi_{b}\right\rangle\right)$, where $\phi_{1}$ is a central automorphism defined by $\phi_{1}(a)=a$ and $\phi_{1}(b)=b^{-1}$. This shows that $\operatorname{Aut}(H) \cong S_{3} \times C_{2}$. In case $H=C_{5} \rtimes C_{4}=\langle a\rangle \rtimes\langle b\rangle$ we have that $\operatorname{Aut}(H) \cong$ $\left\langle\phi_{1}\right\rangle \times\left(\left\langle\phi_{a}\right\rangle \rtimes\left\langle\phi_{b}\right\rangle\right)$, where $\phi_{1}$ is a central automorphism defined by $\phi_{1}(a)=a$ and $\phi_{1}(g)=b^{-1}$. This shows that $\operatorname{Aut}(H) \cong C_{2} \times\left(C_{5} \rtimes C_{4}\right)$, with $C_{4}$ acting faithfully on $C_{5}$.

If $H=C_{3} \rtimes C_{4}<G$ then its image in $A_{5}$ is $S_{3}$ and hence there exists $C_{2}<S_{5}$ such that $S_{3} \times C_{2}<S_{5}$. It follows that the latter subgroup of $S_{5}$ is the image of a subgroup $K$ containing $H$ and such that $K \cong C_{3} \rtimes K_{8}$. From this we have that $\phi_{1} \in \operatorname{Aut}(H)$ is induced by the conjugation of an element of $K_{8}<K$. Since inner automorphisms account for $S_{3}$, it follows that all automorphisms of $H$ are induced by conjugation with an element of $G_{0}$. However, if $H=C_{5} \rtimes C_{4}<G$ then we have that $\operatorname{Aut}\left(S L(2,5) \cong S_{5}\right.$ must have a section of order $|\operatorname{Aut}(H)|=40$, which is not the case. It is not hard to see that $\phi_{1}$ is an automorphism, which cannot be extended to the whole group.

ACKNOWLEDGEMENTS. The second author was supported in part by Onderzoeksraad of Vrije Universiteit Brussel and Fonds voor Wetenschappelijk Onderzoek (Belgium). The third author is grateful to the Departamento de Matemática of the Universidade Federal of Paraíba, Brazil, for financial support and its warm hospitality. The research of the third author was supported by CNPq-Brazil and FAPESP-Brazil Proc. 2008/57930-1. The last author is grateful to the University of São Paulo for its warm hospitality. The research of the last author was supported by CNPq-Brazil and FAPESP-Brazil Proc. 2008/57930-1.

\section{REFERENCES}

1. A. C. P Azevedo, G. G. Bastos and S. O. Juriaans, Extension of automorphisms of subgroups of abelian and polycyclic-by-finite groups, J. Algebr Appl. 6(2) (2007), 315-322. 29-33.

2. D. Bertholf and G. Walls, Finite quasi-injective groups, Glasgow Math. J. 20 (1970),

3. S. Eilenberg and J. C. Moore, Foundations of relative homological algebra, Mem. Amer. Math. Soc. 55 (1965), 1-10.

4. L. Fuchs, Infinite abelian groups I (Academic Press, New York, 1970). 
5. D. Gorenstein, Finite Groups (Harper \& Row, New York - London, 1968).

6. M. Nogin, A short proof of Eilenberg and Moore's theorem, Cent. Eur. J. Math. 5(1) (2007), 201-204.

7. D. Passman, Permutation groups (W. A. Benjamin Inc., New York, 1968).

8. D. J. A. Robinson, A Course in the theory of groups, second ed. (Springer-Verlag, New York, 1996). $249-259$.

9. M. J. Tomkinson, Infinite quasi-injective groups, Proc. Edinburgh Math. Soc. 31 (1988), 